Rev Dor. 2015;16(3):190-4

\title{
Pain assessment through the brief pain inventory in a low socioeconomic level population
}

Caracterização da dor através do inventário breve de dor em população de baixo nível socioeconômico

Gabriel Venas Santos, Ana Shirley Maranhão Vieira, Bruno Teixeira Goes, Renata de Sousa Mota, Abrahão Fontes Baptista,Kátia Nunes Sá

Page
190

Read

Ana Shirley Vieira Maranhão

Ana Shirley Maranhão Vieira

The authors 